\title{
Tetrahydrocannabinol Sensing in Complex Biofluid with Portable Raman Spectrometer Using Diatomaceous SERS Substrates
}

\author{
Kundan Sivashanmugan ${ }^{1}$, Yong Zhao ${ }^{2}$ and Alan X. Wang ${ }^{1, *}$ \\ 1 School of Electrical Engineering and Computer Science, Oregon State University, Corvallis, OR 97331, USA; \\ sivashak@oregonstate.edu \\ 2 School of Electrical Engineering, The Key Laboratory of Measurement Technology and Instrumentation of \\ Hebei Province, Yanshan University, Qinhuangdao 066004, China; zhaoyong@ysu.edu.cn \\ * Correspondence: wang@eecs.oregonstate.edu
}

Received: 16 September 2019; Accepted: 11 October 2019; Published: 14 October 2019

\begin{abstract}
Using thin-layer chromatography in tandem with surface-enhanced Raman spectroscopy (TLC-SERS) and tetrahydrocannabinol (THC) sensing in complex biological fluids is successfully conducted with a portable Raman spectrometer. Both THC and THC metabolites are detected from the biofluid of marijuana-users as biomarkers for identifying cannabis exposure. In this article, ultra-sensitive SERS substrates based on diatomaceous earth integrated with gold nanoparticles (Au NPs) were employed to detect trace levels of cannabis biomarkers in saliva. Strong characteristic THC and THC metabolite SERS peaks at 1601 and $1681 \mathrm{~cm}^{-1}$ were obtained despite the moderate interference of biological molecules native to saliva. Urine samples were also analyzed, but they required TLC separation of THC from the urine sample to eliminate the strong influence of urea and other organic molecules. TLC separation of THC from the urine was performed by porous microfluidic channel devices using diatomaceous earth as the stationary phase. The experimental results showed clear separation between urea and THC, and strong THC SERS characteristic peaks. Principal component analysis (PCA) was used to analyze the SERS spectra collected from various THC samples. The spectra in the principal component space were well clustered for each sample type and share very similar scores in the main principal component (PC1), which can serve as the benchmark for THC sensing from complex SERS spectra. Therefore, we proved that portable Raman spectrometers can enable an on-site sensing capability using diatomaceous SERS substrates to detect THC in real biological solutions. This portable THC sensing technology will play pivotal roles in forensic analysis, medical diagnosis, and public health.
\end{abstract}

Keywords: tetrahydrocannabinol; portable Raman spectrometer; thin layer chromatography; surface-enhanced Raman spectroscopy; photonic crystals

\section{Introduction}

Cannabis-based drug abuse is rapidly increasing worldwide and is closely associated with violence and many other criminal activities [1-5]. The most prominent chemical compound in the Cannabis sativa L. plant is $\Delta^{9}$-tetrahydrocannabinol (THC), which is responsible for the highest degree of psychoactive activity [1-5]. The ability to sense a low concentration $(\mathrm{ng} / \mathrm{mL})$ of THC in biological fluids (e.g., blood, saliva, and urine) within the period of physiological activity would have significant values for potential forensic and medical applications [1-5]. The concentration of THC rapidly reduces with respect to time due to the metabolic breakdown of biological fluids. For instance, after smoking marijuana, the THC concentration in saliva reduces from 148 to $82.6 \mathrm{ng} / \mathrm{L}$ within $30 \mathrm{~min}$. Similarly, the THC concentration in blood samples decreases from 20 to $5 \mathrm{ng} / \mathrm{mL}$ after $24 \mathrm{~h}$ [1-7]. 
Numerous efforts have been employed to detect THC in biofluids, including gas chromatography-mass spectrometry (GC-MS), high-performance liquid chromatography (HPLC), and enzyme-linked immunosorbent assay $[3,4,8-11]$. These sensing techniques usually require complex sample preparation or time-consuming, expensive procedures. Thus, there is an urgent need for an alternative, rapid, and sensitive analytical technique to accurately perceive THC in biological fluids. Surface-enhanced Raman scattering (SERS) has been increasingly adopted for chemical and biological sensing of various species at very low concentrations due to the extraordinary enhancement factors (EFs) from plasmonic nanostructures [12-14]. In recent years, SERS techniques have also been applied to illicit drug detection [15-19]. For instance, our group used hybrid photonic crystal-plasmonic SERS substrates, which were fabricated by depositing silver nanoparticles (Ag NPs) into diatom photonic crystals $[13,20]$. We successfully detected THC in spiked saliva samples, which achieved a detection limit of $10^{-12} \mathrm{M}$, by using a confocal Raman microscope. However, using a laboratory bench-top Raman microscope is unsuitable for on-site detection or portable sensing. In addition, although direct SERS sensing of THC from saliva is feasible, many ordinary biomolecules (e.g., protein and urea acid) or particles will disrupt direct THC SERS sensing from complex biofluid samples such as urine or blood. These biomolecules have high binding affinity to SERS substrates and induce severe interferences with strong background noise [12,21-23]. Therefore, it is necessary to develop a chromatography technique in tandem with SERS sensing to detect THC from real complex biofluids.

Compared with traditional GC or HPLC, thin-layer chromatography (TLC) is intrinsically suitable for portable sensing due to its exclusive advantages in a simple equipment requirement, rapid processing time, and cost effectiveness $[4,8,21]$. Recently, TLC in tandem with a SERS (TLC-SERS) method has been developed for various sensing applications [21,24-28]. Our group also demonstrated TLC-SERS sensing using diatomaceous earth substrates for food safety and illicit drug detection with ultra-high detection sensitivity [24-27]. Diatomaceous earth consists of fossilized remains of diatoms, which are hard-shelled algae $[24,25,29,30]$. Diatomaceous earth particles possess unique material properties in terms of high porosity, large surface area, strong adsorption capacity, and abundant hydroxyl groups at the bio-silica surface $[24,25,29]$. The two-dimensional (2D) periodic pores embedded within the diatom shells resemble nanoscale photonic crystal features, which can provide additional SERS EFs [24,25]. In this article, we report patterned porous diatomaceous channels as the stationary phase for separating THC from urine samples. By adding gold nanoparticles (Au NPs) onto the diatomaceous substrates, SERS sensing was conducted via a portable Raman spectrometer. Such diatomaceous TLC-SERS technique achieved $10 \mathrm{ppm}$ THC sensing from spiked urine samples and clear SERS spectra from urine of volunteer marijuana users.

\section{Materials and Methods}

\subsection{Synthesis of Gold Nanoparticles}

The $\mathrm{Au}$ NPs were synthesized by a sodium citrate reduction method. In addition, $100 \mathrm{~mL}$ of aqueous solution containing $1 \mathrm{mM}$ chloroauric acid was heated to a near-boiling temperature under vigorous stirring. Five $\mathrm{mL}$ of $1 \%$ sodium citrate solution was then added to the boiling solution and the final reaction continued for an additional hour. The final mixture solution was heated until the color of the solution became reddish-brown. The final solution was then cooled to room temperature and washed three times by deionized water to remove impurities. The Au NPs were then collected for SERS sensing.

\subsection{Fabrication of Porous Diatomaceous TLC Plates}

The diatomaceous earth powder was dried at $160^{\circ} \mathrm{C}$ for $12 \mathrm{~h}$ in an oven and cooled to room temperature. Subsequently, $6 \mathrm{~g}$ of the powder was dispersed in $10 \mathrm{~mL}$ of $0.4 \%$ aqueous solution of carboxymethyl cellulose under vigorous stirring. The glass slides were first covered with an adhesive tape. Next, arrayed slits were cut through the tape at different widths using a sharp razor blade. 
Afterward, the mixed solution was deposited onto the patterned glass substrate by spin-coating at 800 rpm for $10 \mathrm{~s}$ and the tape was then removed. The diatomaceous plates were dried in a dark place and baked at $110{ }^{\circ} \mathrm{C}$ for $3 \mathrm{~h}$ to increase the adhesion to the glass surface.

\subsection{Biofluid Samples Preparation}

Saliva samples were collected directly after (i.e., within $15 \mathrm{~min}$ of) marijuana use from volunteers. The collected saliva samples $(2 \mathrm{~mL})$ were mixed with an equal amount of water and centrifuged at $8000 \mathrm{rpm}$ for $15 \mathrm{~min}$ to remove oral impurities and food residues. Then, the saliva samples were stored at $4{ }^{\circ} \mathrm{C}$ to suppress degradation. For SERS measurements, the as-prepared saliva samples (2 $\mu \mathrm{L})$ were measured using diatomaceous SERS substrates. The urine samples $(15 \mathrm{~mL})$ were collected from both non-marijuana users and marijuana users in glass vials in the morning for multiple days. The urine samples from the non-marijuana volunteers were mixed with various concentrations of THC (1000 ppm to $10 \mathrm{ppm}$ ) for SERS characterization. The urine samples from marijuana-users and THC-spiked urine samples were filtered with syringe filters and diluted with water for the SERS measurement. All samples were analyzed within $3 \mathrm{~h}$ of collection.

\subsection{TLC-SERS Method}

The TLC-SERS detection was designed to detect THC in urine samples, as shown in Figure 1a. To separate THC from the urine solution, $2 \mu \mathrm{L}$ of the urine sample was drop-cast on the TLC plate (i.e., $12 \mathrm{~mm}$ from the edge of the plate) and air-dried. Next, the TLC plate with the urine sample was immersed in the development chamber with the mobile-phase eluent (chloroform-acetone-ammonium hydroxide, 40/20/40 v/v), which migrates along the porous diatomaceous channels driven by the capillary force [24,25]. After THC was separated from other species, the TLC plate was removed from the development chamber and air-dried, as shown in Figure 1a. The isolated THC was visualized using fast blue salt colorimetry. Subsequently, $2 \mu \mathrm{L}$ of Au NPs in solution were directly dropped onto the separation spot and measured by the portable Raman spectrometer.

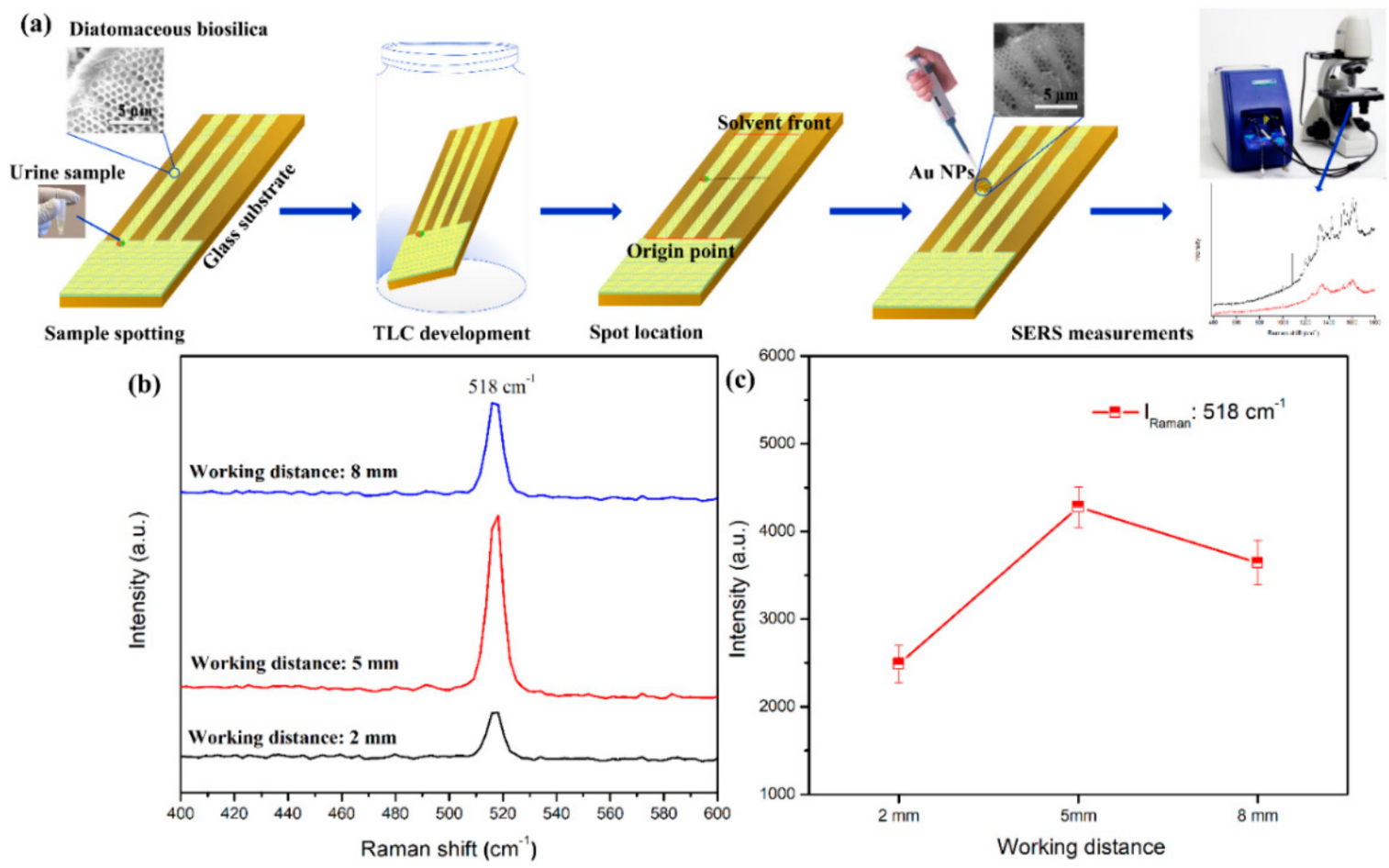

Figure 1. (a) Process of TLC-SERS detection of THC from urine samples via a portable Raman spectrometer. (b) The Raman spectra of a Si wafer at various working distances from the fiber tip, and (c) intensities versus different working distances using the $\mathrm{I}_{\text {Raman }}$ peak at $518 \mathrm{~cm}^{-1}$. 


\subsection{Portable Raman Spectrometer}

The SERS spectra were acquired by a portable Raman spectrometer (B\&W TEK, i-Raman plus, USA) as indicated in Figure 1a. Before the SERS measurement, the working distance of the portable Raman spectrometer was optimized with a Si wafer ranging from 2 to $8 \mathrm{~mm}$. Figure $1 \mathrm{~b}$ illustrates the Raman spectra of different working distances from the Raman probe tip to the Si wafer. The Raman peak of the $\mathrm{Si}$ at $518 \mathrm{~cm}^{-1}$ was used to optimize the working distance, which shows that $5-\mathrm{mm}$ is the optimal working distance. This is demonstrated in Figure 1c. Subsequently, all SERS measurements were conducted at a 5-mm working distance using a $420 \mathrm{~mW}$ and $785 \mathrm{~nm}$ wavelength excitation laser. The laser spot size is $85 \mu \mathrm{m}$ in diameter. The SERS spectra were acquired using 10-s integration time in the spectral range from 400 to $1800 \mathrm{~cm}^{-1}$. The SERS spectra was averaged from 20 consecutive measurements.

\subsection{Enhancement Factor Calculation}

The EF was calculated according to the formula below.

$$
E F=\frac{I_{S E R S} \times N_{B u l k}}{I_{B u l k} \times N_{S E R S}}
$$

where $I_{B u l k}$ and $I_{S E R S}$ are the peak intensity of the normal Raman measurement with $10^{-3} \mathrm{M} \mathrm{THC}$ solution and SERS measurement with $10^{-7} \mathrm{M}$ THC solution, respectively. $N_{B u l k}$ and $N_{\text {SERS }}$ are the number of THC molecules within the laser spot of the portable Raman spectrometer for the normal Raman measurement and SERS measurement, respectively. $N_{\text {Bulk }}$ and $N_{S E R S}$ was calculated using the equation below.

$$
N_{B u l k}=C_{0} \times V_{0} \times N_{A} \times\left(\frac{d}{D_{0}}\right)^{2}
$$

where $C_{0}$ is the concentration of THC for the normal Raman measurement, $V_{0}$ is the volume of THC solution for a normal Raman measurement, $N_{A}$ is the Avogadro constant, $d$ is the laser spot area, and $D_{0}$ is the diameter of the liquid spot in which the THC molecule is distributed on the glass substrate.

$$
N_{S E R S}=C_{S E R S} \times V_{S E R S} \times N_{A} \times\left(\frac{d}{D_{S E R S}}\right)^{2}
$$

where $C_{S E R S}$ is the concentration of the THC for the SERS measurement, $V_{S E R S}$ is the volume of THC solution for the SERS measurement, and $D_{S E R S}$ is the diameter of the liquid spot in which the THC molecule is distributed on the diatomite substrate.

\section{Results and Discussion}

\subsection{Characterization of Plasmonic Photonic Materials}

Field-emission scanning electron microscopy (FE-SEM) images of diatomaceous earth on glass substrates are shown in Figure 2a. The commercial diatomaceous particles are mostly dish-shaped with $2 \mathrm{D}$ periodic pores, which can generate strong guided-mode resonance [24,25,27]. The diatomaceous particles' size ranges from 10 to $25 \mu \mathrm{m}$. The diatomaceous film deposited on the glass substrate works as the stationary phase for the TLC plate. The Au NPs were synthesized using a simple reduction method [22]. Figure $2 b$ depicts the ultraviolet-visible absorption spectrum of the Au NPs. The surface plasmon resonance peak was located at $521 \mathrm{~nm}$ with a narrow bandwidth, which suggests that the NPs were mono-dispersed in the aqueous solution without significant aggregation. The FE-SEM image of the Au NPs was presented in the inset of Figure 2b, which demonstrated an average diameter of $\sim 60 \mathrm{~nm}$ and exhibited a nearly spherical shape. After TLC separation, $5 \mu \mathrm{L}$ of the Au NP solution 
was drop-casted onto the THC aggregation spot on the TLC chip. The distribution of Au NPs on the surface of the diatomaceous particle is illustrated in Figure 2c.
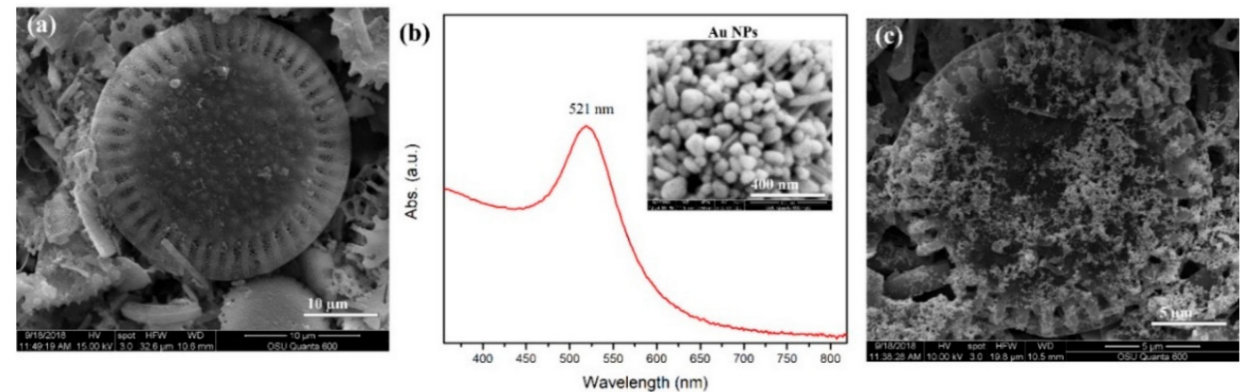

Figure 2. (a) The FE-SEM top-view image of diatomaceous particles. (b) The SPR spectra of as-prepared $\mathrm{Au}$ NPs with an inset FE-SEM image of Au NPs, and (c) the FE-SEM top-view image of Au NPs on the diatomaceous particle.

\subsection{SERS Analyses of THC in Methanol and Saliva Samples}

Various concentrations of THC (1-1000 ppm) in methanol solution were dispensed on the diatomaceous films, and then Au NPs were drop-casted for SERS analysis. The weak Raman signals were observed from pure THC solution (1000 ppm) on the glass substrate due to the low THC Raman activity. The SERS spectra of THC at various concentrations were examined using the $785 \mathrm{~nm}$ portable Raman spectrometer, as demonstrated in Figure 3a. The SERS bands of THC are located at 958, 1001, $1091,1201,1287,1342,1427,1482,1565$, and $1601 \mathrm{~cm}^{-1}$, and their assigned bands are enumerated in Table $1[15-17,20]$. The most intense SERS peak appeared at $1601 \mathrm{~cm}^{-1}$, which is assigned to the C-C stretching mode. The EF for Au NPs on diatomite substrate and pure Au NPs was compared using the strong SERS band at $1601 \mathrm{~cm}^{-1}$. Au NPs on diatomite substrate showed the highest EF $\left(1.4 \times 10^{6}\right)$ compared to pure Au NPs $\left(1.2 \times 10^{2}\right)$ due to the strong local field that occurred among the congregated $\mathrm{Au}$ NPs inside the diatomite pores.
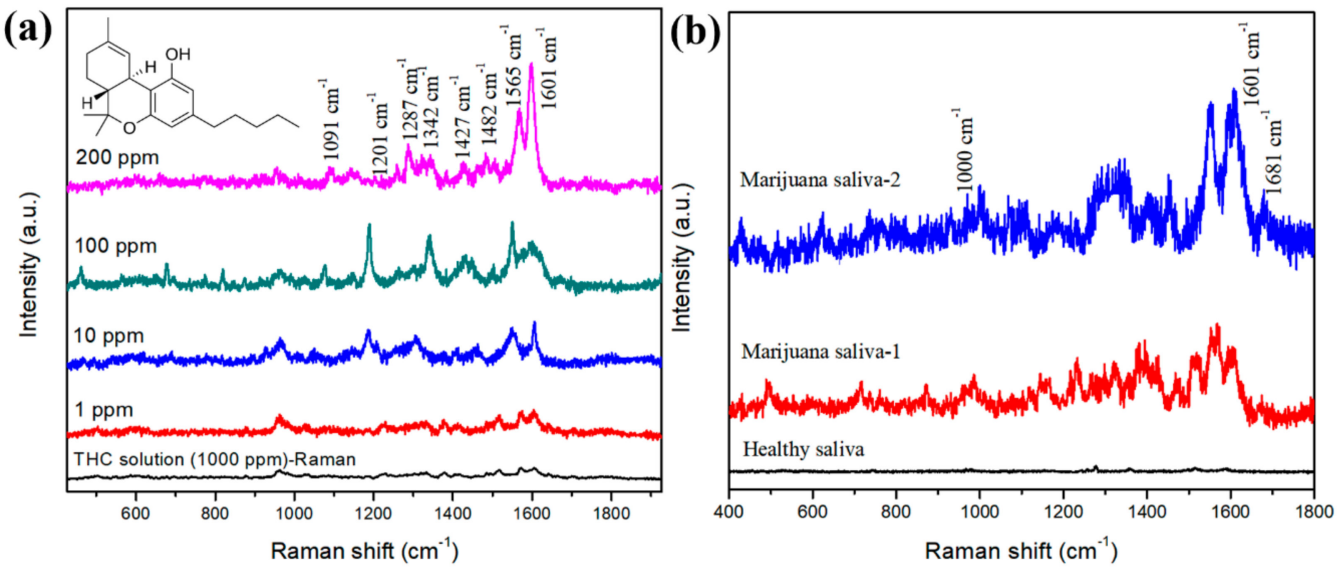

Figure 3. (a) SERS spectra of THC molecules (200, 100, 10, and 1 ppm) in methanol, and (b) SERS spectra from marijuana-users' saliva samples. 
Table 1. Assignment of Raman peaks for urea and THC molecules.

\begin{tabular}{|c|c|c|}
\hline \multicolumn{2}{|c|}{ Raman Shifts $\left(\mathrm{cm}^{-1}\right)$} & \multirow{2}{*}{ Raman Bands Assignments } \\
\hline Urea & THC & \\
\hline 627,727 & & $\mathrm{C}-\mathrm{H}$ bending vibration \\
\hline 780 & & C-C stretching vibration \\
\hline 830 & & C-C stretching vibration \\
\hline 903,937 & 932,958 & $\mathrm{C}-\mathrm{O}-\mathrm{H}$ stretching vibration \\
\hline \multirow[t]{5}{*}{1000,1038} & & N-C-N stretching vibration \\
\hline & 998,1001 & C-C stretching vibration \\
\hline & 1091 & C-C stretching vibration \\
\hline & $1153,1164,1171,1192$ & C-C stretching vibration \\
\hline & $1201,1210,1250$ & $\mathrm{CH}$ deformation \\
\hline \multirow[t]{2}{*}{$1245,1256,1293$} & & $\mathrm{H}-\mathrm{C}-\mathrm{H}$ bending vibration \\
\hline & $1343,1366,1367$ & C-H deformation \\
\hline \multirow[t]{5}{*}{1382,1407} & 1384,1392 & $\mathrm{H}-\mathrm{C}-\mathrm{H}$ bending vibration \\
\hline & $1448,1475,1512$ & -C-H deformation \\
\hline & 1582,1584 & C-C stretching \\
\hline & $1600-1605$ & C-C stretching \\
\hline & 1681 & $\mathrm{O}-\mathrm{C}=\mathrm{O}$ stretching \\
\hline
\end{tabular}

Marijuana-users' saliva samples were also used to detect THC using the diatomaceous SERS substrate. Figure $3 b$ presents the THC SERS spectra of two marijuana-users' saliva samples. Both saliva samples clearly indicated the THC SERS characteristic peak at $1601 \mathrm{~cm}^{-1}$, while other THC SERS peaks were not prominent. The SERS bands of THC in marijuana-users' samples are located at $1000,1067,1230,1345,1409,1454,1548,1601$, and $1681 \mathrm{~cm}^{-1}$, and are assigned to their respective bonds in Table 1. The THC metabolite SERS peak appeared at $1681 \mathrm{~cm}^{-1}$, which is assigned to the $\mathrm{O}-\mathrm{C}=\mathrm{O}$ and indicate the formation of the THC metabolite [20]. In human biological fluids, the THC structure can be modified and form a hydroxyl metabolite and a carboxylic acid metabolite due to its rapid oxidization, which can be found after a few days of intake of THC $[1,3,20]$. It shows that, in the case of healthy volunteer saliva, the biomolecules present very little interference to the SERS spectrum and introduces acceptable background noise. Therefore, the THC in saliva is directly detected using SERS due to the absence of a significant biomolecule or particle interference.

\subsection{THC Sensing in Urine Samples Using TLC-SERS}

In the case of a complex solution such as plasma or urine samples, chromatography methods are required for separating THC from other biomolecules. The collected urine samples were directly measured using SERS, as shown in Figure 4a. The SERS peaks from urine samples were mostly related to urea Raman bands, which are enumerated in Table 1 [20,31-33]. Therefore, direct SERS sensing of THC in urine samples, either from artificially spiked urine or obtained from a volunteer marijuana user, was not successful due to the strong background interference from urea, which usually has strong binding affinity to the SERS substrate.

Chromatography separation of THC in the urine sample was required for practical sensing applications. Chloroform-acetone-ammonium hydroxide $(40 / 20 / 40 v / v)$ eluent was used as the mobile-phase eluent. The widths of the TLC plate channels were 1,2, and $3 \mathrm{~mm}$, respectively, as shown in the inset in Figure $4 \mathrm{~b}$. After TLC separation, SERS spectra of $500 \mathrm{ppm}$ THC were measured from various widths of TLC channels, as shown in Figure $4 \mathrm{~b}$. The SERS bands of THC are located at 998, $1001,1153,1250,1342,1366,1448,1482$, and $1605 \mathrm{~cm}^{-1}$, which match the SERS spectra of pure THC solution. This is well-summarized in Table $1[15-17,20]$. Nonetheless, the strongest SERS characteristic peak is located at $1605 \mathrm{~cm}^{-1}$, which is assigned to the $C-C$ stretching modes. The smallest width $(1 \mathrm{~mm})$ TLC channel produced the strongest SERS signals when compared to the other channels due to the superior THC confinement. Therefore, all TLC-SERS sensing measurements in this study were based on a 1-mm channel. 

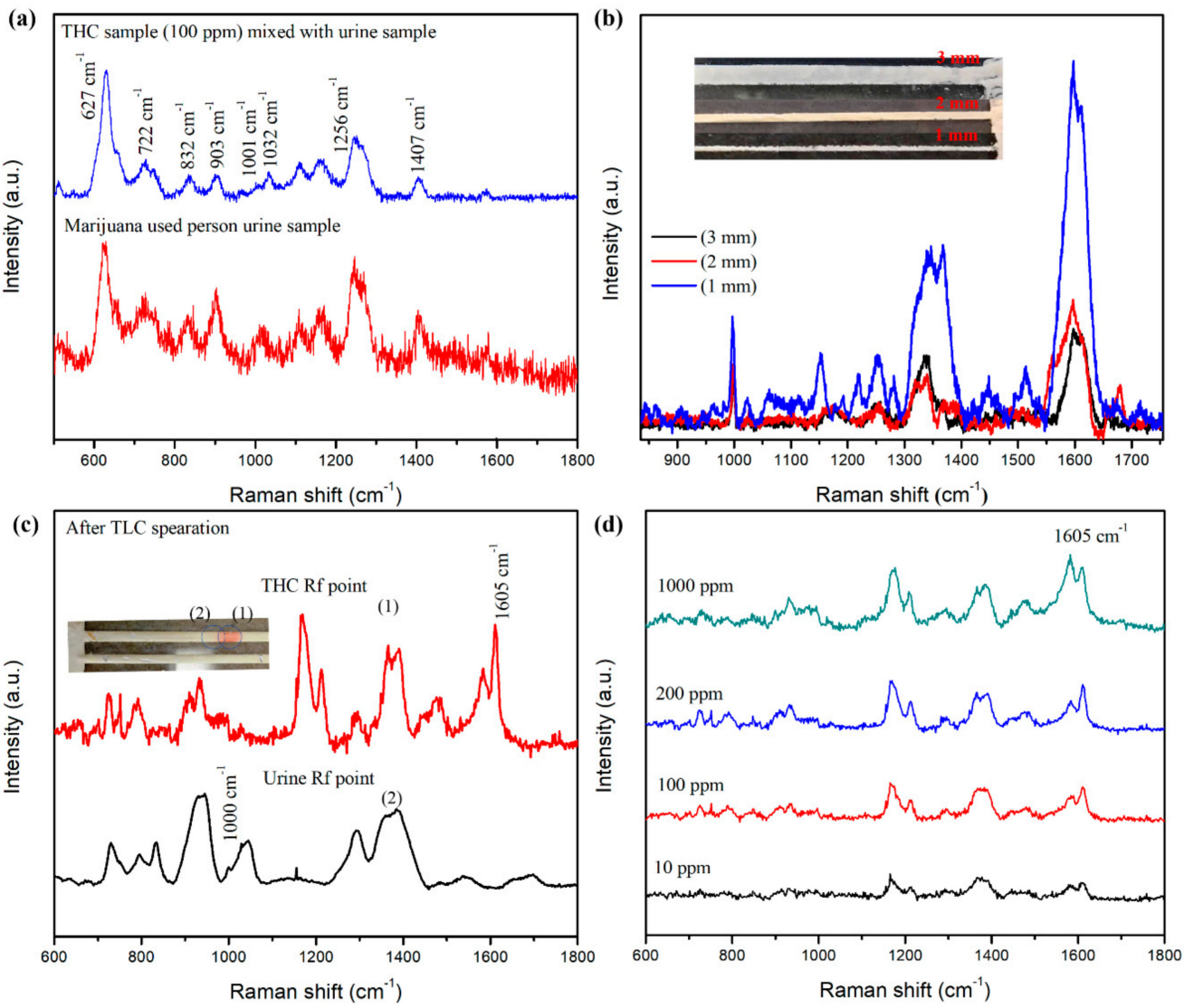

Figure 4. (a) SERS spectra of THC-spiked (100 ppm) urine, and marijuana-user's urine samples measured from the diatomaceous SERS substrates. (b) TLC-SERS spectra of THC (500 ppm) from diatomaceous earth TLC plates with 1,2, and 3-mm channel widths (inset figure shows TLC channels). (c) TLC-SERS spectra of THC and urea from THC-spiked urine sample, and (d) TLC-SERS spectra of THC from various concentrations of THC-spiked urine samples.

The resulting clear separation of THC in the THC-spiked urine sample is illustrated in the inset in Figure 4c. A quick migration of the THC on the diatomaceous earth TLC plate was observed and can be attributed to the molecular polarity of THC. The obtained retention factor (Rf), which is calculated as the ratio of the distance migrated by the target analyte to the distance migrated by the eluent solvent, was 0.69 and $0.72 \mathrm{~cm}$ for the concentrated urea and THC, respectively, which was shown in the inset of Figure 4c. The SERS spectra at two marked positions demonstrated strong SERS characteristic peaks of urea (1) and THC (2), as illustrated in Figure 4c. These results confirmed the THC separation from urine samples using the diatomaceous TLC plate. Next, THC at various concentrations (i.e., $1000 \mathrm{ppm}$ to $10 \mathrm{ppm}$ ) was artificially added to urine solutions. Urine samples were collected from non-marijuana using volunteers in the morning. After the separation of THC from these artificially-contaminated urine samples, SERS spectra were measured on the THC spots, according to the Rf value. The strongest SERS peak of THC appeared at $1605 \mathrm{~cm}^{-1}$, as shown in Figure $4 \mathrm{~d}$. There are several clear urea Raman peaks at $627,727,780,830,903,937,1000,1038,1256,1293,1382$, and $1407 \mathrm{~cm}^{-1}$ and THC Raman peaks at $722,785,932,1164,1210,1367,1392,1478,1582$, and $1605 \mathrm{~cm}^{-1}$. Their assigned bands are listed in Table 1. Compared with direct THC SERS sensing from saliva samples, more THC-related Raman peaks were obtained using TLC separation. This indicated a clear separation of THC from complex samples.

To demonstrate THC sensing from real biological fluid, TLC-SERS sensing from marijuana-user's urine samples was conducted. Before TLC separation, only urea SERS peaks can be measured and the 1165,1245 , and $1407 \mathrm{~cm}^{-1}$ Raman peaks of urea were clearly observed, as illustrated in 
Figure 5. After TLC separation, the SERS peak of THC at 1003 and $1603 \mathrm{~cm}^{-1}$ can be clearly observed. However, relatively few THC-related Raman peaks were obtained when compared with THC-spiked urine samples. This reflects the strong impact of the marijuana user's urine background from other biomolecules interference.

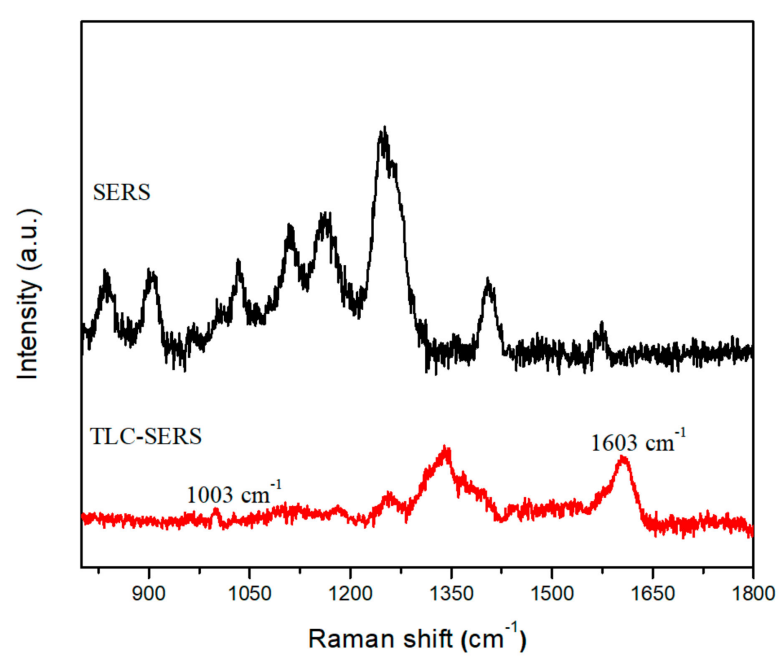

Figure 5. SERS spectra of THC in a marijuana-user urine sample before and after TLC separation.

In order to quantitatively evaluate the effectiveness of our portable SERS sensing technique, we used Principal component analysis (PCA), which is a multivariate statistical method for feature extraction, to analyze the samples we measured in our previous studies. PCA was performed using math-works (MATLAB) software based on 20 spectra for each sample type. PCA was applied to six groups of data: SERS spectra without TLC (i.e., pure THC, healthy people's saliva and marijuana user's saliva) and with TLC separation (i.e., urine sample, THC-spiked urine sample, and marijuana user's urine sample), as shown in Figure 6. In this analysis, the first three PCs of the SERS spectra account for PC1 (98.25\%), PC2 (0.75\%), and PC3 (0.50\%) of the variance for the six groups of samples, as displayed in Figure 6. It is very clear that PC1 is the main principle component and should account for the major score. When comparing pure THC (200 ppm) and marijuana users' saliva samples using the SERS-only sensing method, the PCA plot shows highly congregated clusters arranged in close proximity to each other. The PCA results indicated that saliva samples contained less molecular interference and the results closely matched pure THC solution. After TLC separation, each sample matrix formed a good cluster but slightly spread out along a single plane. The samples containing THC have a similar PC1 score compared to pure THC solution, while the urine samples and healthy people's saliva without THC are far away from other samples and have a very different PC1 score. This indicates the significant difference of the SERS spectra due to the presence of THC in different biological samples. When comparing THC-spiked urine samples and marijuana user's urine samples, the latter are further away from the pure THC, which suggests stronger background interference and matches our previous analysis. Therefore, using the PC1 score from the PCA results can effectively evaluate the presence of THC in complex biological samples. 


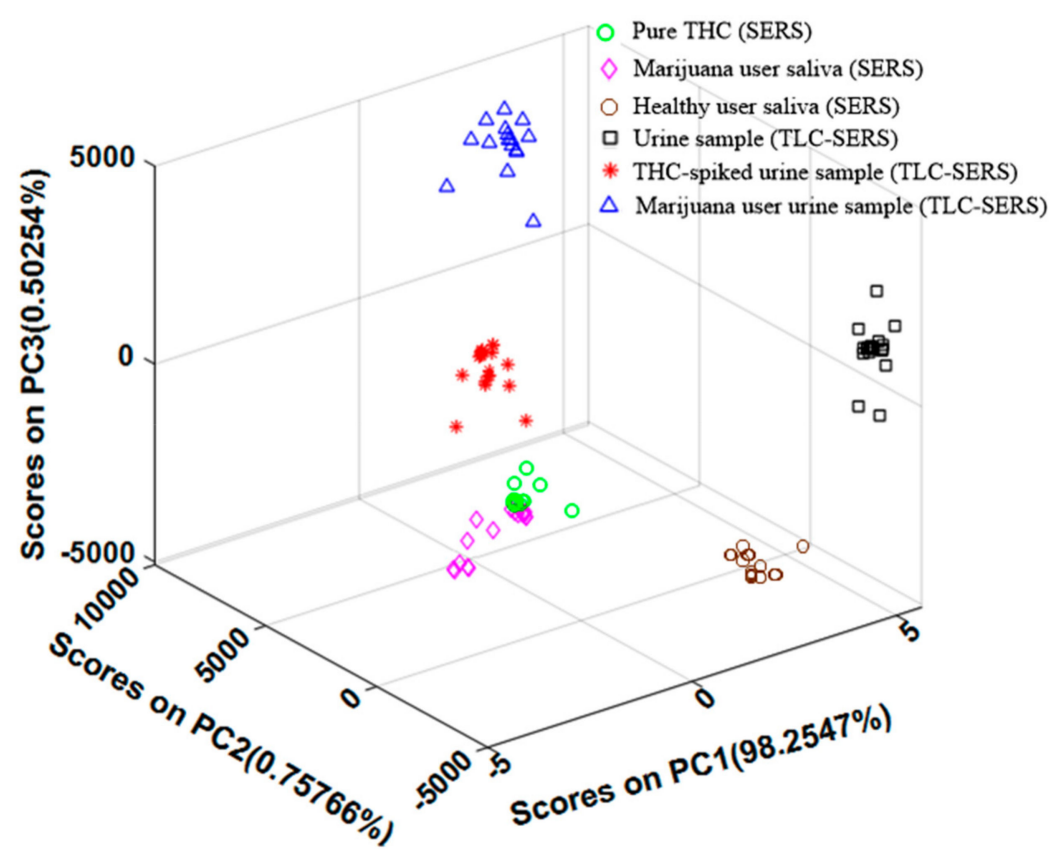

Figure 6. PCA scatter plot of SERS spectra of the first three PC score: pure THC (SERS), marijuana user saliva (SERS), healthy people saliva, urine sample (TLC-SERS), THC-spiked urine sample (TLC-SERS), and marijuana user's urine sample (TLC-SERS).

\section{Conclusions}

In this article, we presented a THC-sensing technique using a portable Raman spectrometer to detect THC in the saliva and urine samples of marijuana users. With the high-performance diatomaceous SERS substrates, we obtained the SERS spectra of THC in saliva without pre-treating the samples. To sense THC from more complex biofluids, we developed a TLC-SERS sensing protocol to separate and detect THC molecules from urine samples of marijuana users based on porous diatomaceous microfluidic channels as the stationary phase. The described THC detection method allows for rapid and sensitive detection of THC analyte in spiked urine with concentrations ranging from 1000 to $10 \mathrm{ppm}$. Detection of THC in the marijuana user's urine sample was clearly obtained after TLC separation. Furthermore, PCA was used to analyze the SERS spectra of all samples and shows a clear difference of THC from the complex background. In summary, such a portable SERS-sensing technique can play a pivotal role in future forensic and medical applications.

Author Contributions: K.S. and A.X.W. conceived and designed the experiments; K.S performed the experiments; K.S. and Y.Z. analyzed the data; K.S. and A.X.W. wrote the manuscript.

Funding: This research was funded by the National Institutes of Health, the Unites States Department of Agriculture, and the National Science Foundation.

Acknowledgments: The National Institutes of Health under Grant No. 1R21DA0437131, the Unites States Department of Agriculture under Grant No. 2017-67021-26606, and the National Science Foundation under Grant No. 1701329 financially supported this work.

Conflicts of Interest: The authors declare no conflict of interest.

\section{References}

1. Murray, R.M.; Morrison, P.D.; Henquet, C.; Forti, M.D. Cannabis, the mind and society: The hash realities. Nat. Rev. Neurosci. 2007, 8, 885. [CrossRef] [PubMed]

2. Kalant, H. Medicinal Use of Cannabis: History and Current Status. Pain Res. Manag. 2001, 6. [CrossRef] [PubMed] 
3. Grauwiler, S.B.; Scholer, A.; Drewe, J. Development of a LC/MS/MS method for the analysis of cannabinoids in human EDTA-plasma and urine after small doses of Cannabis sativa extracts. J. Chromatogr. B 2007, 850, 515-522. [CrossRef]

4. Wohlfarth, A.; Mahler, H.; Auwärter, V. Rapid isolation procedure for $\Delta 9$-Tetrahydrocannabinolic acid A (THCA) from Cannabis sativa using two flash chromatography systems. J. Chromatogr. B 2011, 879, 3059-3064. [CrossRef] [PubMed]

5. Sultan, S.; Millar, S.; O'Sullivan, S.; England, T. A Systematic Review and Meta-Analysis of the In Vivo Haemodynamic Effects of $\Delta$ 9-Tetrahydrocannabinol. Pharmaceuticals 2018, 11, 13. [CrossRef] [PubMed]

6. Karschner, E.L.; Schwilke, E.W.; Lowe, R.H.; Darwin, W.D.; Herning, R.I.; Cadet, J.L.; Huestis, M.A. Implications of plasma Delta9-tetrahydrocannabinol, 11-hydroxy-THC, and 11-nor-9-carboxy-THC concentrations in chronic cannabis smokers. J. Anal. Toxicol. 2009, 33, 469-477. [CrossRef] [PubMed]

7. Milman, G.; Schwope, D.M.; Gorelick, D.A.; Huestis, M.A. Cannabinoids and metabolites in expectorated oral fluid following controlled smoked cannabis. Clin. Chim. Acta 2012, 413, 765-770. [CrossRef]

8. Vinson, J.A.; Patel, D.D.; Patel, A.H. Detection of tetrahydrocannabinol in blood and serum using a fluorescent derivative and thin-layer chromatography. Anal. Chem. 1977, 49, 163-165. [CrossRef]

9. Scheidweiler, K.B.; Desrosiers, N.A.; Huestis, M.A. Simultaneous quantification of free and glucuronidated cannabinoids in human urine by liquid chromatography tandem mass spectrometry. Clin. Chim. Acta 2012, 413, 1839-1847. [CrossRef]

10. Lurie, I.S.; Meyers, R.P.; Conver, T.S. Capillary Electrochromatography of Cannabinoids. Anal. Chem. 1998, 70, 3255-3260. [CrossRef]

11. Abraham, T.T.; Lowe, R.H.; Pirnay, S.O.; Darwin, W.D.; Huestis, M.A. Simultaneous GC-EI-MS determination of Delta9-Tetrahydrocannabinol, 11-hydroxy-Delta9-tetrahydrocannabinol, and 11-nor-9carboxy-Delta9-tetrahydrocannabinol in human urine following tandem Enzyme-Alkaline hydrolysis. J. Anal. Toxicol. 2007, 31, 477-485. [CrossRef] [PubMed]

12. Luo, S.-C.; Sivashanmugan, K.; Liao, J.-D.; Yao, C.-K.; Peng, H.-C. Nanofabricated SERS-active substrates for Single-Molecule to virus detection in vitro: A review. Biosens. Bioelectron. 2014, 61, 232-240. [CrossRef] [PubMed]

13. Sivashanmugan, K.; Squire, K.; Kraai, J.A.; Tan, A.; Zhao, Y.; Rorrer, G.L.; Wang, A.X. Biological Photonic Crystal-Enhanced Plasmonic Mesocapsules: Approaching Single-Molecule Optofluidic-SERS Sensing. Adv. Opt. Mater. 2019, 7, 1900415. [CrossRef]

14. Kim, K.; Guo, J.; Liang, Z.; Fan, D. Artificial Micro/Nanomachines for Bioapplications: Biochemical Delivery and Diagnostic Sensing. Adv. Funct. Mater. 2018, 28, 1705867. [CrossRef]

15. Islam, S.K.; Cheng, Y.P.; Birke, R.L.; Green, O.; Kubic, T.; Lombardi, J.R. Rapid and sensitive detection of synthetic cannabinoids AMB-FUBINACA and $\alpha$-PVP using surface enhanced Raman scattering (SERS). Chem. Phys. 2018, 506, 31-35. [CrossRef]

16. Yüksel, S.; Schwenke, A.M.; Soliveri, G.; Ardizzone, S.; Weber, K.; Cialla-May, D.; Hoeppener, S.; Schubert, U.S.; Popp, J. Trace detection of tetrahydrocannabinol (THC) with a SERS-Based capillary platform prepared by the in situ microwave synthesis of AgNPs. Anal. Chim. Acta 2016, 939, 93-100. [CrossRef]

17. Milliken, S.; Fraser, J.; Poirier, S.; Hulse, J.; Tay, L.-L. Self-Assembled vertically aligned Au nanorod arrays for Surface-Enhanced Raman scattering (SERS) detection of Cannabinol. Spectrochim. Acta Part A Mol. Biomol. Spectrosc. 2018, 196, 222-228. [CrossRef]

18. Gao, J.; Zhang, N.; Ji, D.; Song, H.; Liu, Y.; Zhou, L.; Sun, Z.; Jornet, J.M.; Thompson, A.C.; Collins, R.L.; et al. Superabsorbing Metasurfaces with Hybrid Ag-Au Nanostructures for Surface-Enhanced Raman Spectroscopy Sensing of Drugs and Chemicals. Small Methods 2018, 2, 1800045. [CrossRef]

19. Xu, S.-Q.; Wen, B.-Y.; Zhang, L.-N.; Zhang, H.; Gao, Y.; Nataraju, B.; Xu, L.-P.; Wang, X.; Li, J.-F.; Tian, Z.-Q. Evaluation of cigarette flavoring quality via Surface-Enhanced Raman spectroscopy. Chem. Commun. 2018, 54, 10882-10885. [CrossRef]

20. Sivashanmugan, K.; Squire, K.; Tan, A.; Zhao, Y.; Kraai, J.A.; Rorrer, G.L.; Wang, A.X. Trace Detection of Tetrahydrocannabinol in Body Fluid via Surface-Enhanced Raman Scattering and Principal Component Analysis. ACS Sens. 2019, 4, 1109-1117. [CrossRef]

21. Huang, R.; Han, S.; Li, X.S. Detection of Tobacco-Related biomarkers in urine samples by Surface-Enhanced Raman spectroscopy coupled with Thin-Layer chromatography. Anal. Bioanal. Chem. 2013, 405, 6815-6822. [CrossRef] [PubMed] 
22. Sivashanmugan, K.; Huang, W.-L.; Lin, C.-H.; Liao, J.-D.; Lin, C.-C.; Su, W.-C.; Wen, T.-C. Bimetallic nanoplasmonic Gap-Mode SERS substrate for lung normal and Cancer-Derived exosomes detection. J. Taiwan Inst. Chem. Eng. 2017, 80, 149-155. [CrossRef]

23. Sivashanmugan, K.; Liao, J.-D.; Shao, P.-L.; Haochih Liu, B.; Tseng, T.-Y.; Chang, C.-Y. Intense Raman scattering on hybrid Au/Ag nanoplatforms for the distinction of MMP-9-digested collagen Type-I fiber detection. Biosens. Bioelectron. 2015, 72, 61-70. [CrossRef] [PubMed]

24. Kong, X.; Chong, X.; Squire, K.; Wang, A.X. Microfluidic diatomite analytical devices for illicit drug sensing with ppb-Level sensitivity. Sens. Actuators B Chem. 2018, 259, 587-595. [CrossRef]

25. Kong, X.; Squire, K.; Chong, X.; Wang, A.X. Ultra-Sensitive lab-on-a-chip detection of Sudan I in food using Plasmonics-Enhanced diatomaceous thin film. Food Control 2017, 79, 258-265. [CrossRef]

26. Kong, X.; Squire, K.; Li, E.; LeDuff, P.; Rorrer, G.L.; Tang, S.; Chen, B.; McKay, C.P.; Navarro-Gonzalez, R.; Wang, A.X. Chemical and Biological Sensing Using Diatom Photonic Crystal Biosilica with In-Situ Growth Plasmonic Nanoparticles. IEEE Trans. NanoBiosci. 2016, 15, 828-834. [CrossRef]

27. Tan, A.; Zhao, Y.; Sivashanmugan, K.; Squire, K.; Wang, A.X. Quantitative TLC-SERS detection of histamine in seafood with support vector machine analysis. Food Control 2019, 103, 111-118. [CrossRef]

28. Zhang, Z.-M.; Liu, J.-F.; Liu, R.; Sun, J.-F.; Wei, G.-H. Thin Layer Chromatography Coupled with Surface-Enhanced Raman Scattering as a Facile Method for On-Site Quantitative Monitoring of Chemical Reactions. Anal. Chem. 2014, 86, 7286-7292. [CrossRef]

29. Leonardo, S.; Prieto-Simón, B.; Campàs, M. Past, present and future of diatoms in biosensing. TrAC Trends Anal. Chem. 2016, 79, 276-285. [CrossRef]

30. Yang, W.; Lopez, P.J.; Rosengarten, G. Diatoms: Self assembled silica nanostructures, and templates for bio/chemical sensors and biomimetic membranes. Analyst 2011, 136, 42-53. [CrossRef]

31. Dong, R.; Weng, S.; Yang, L.; Liu, J. Detection and direct readout of drugs in human urine using dynamic Surface-Enhanced Raman spectroscopy and support vector machines. Anal. Chem. 2015, 87, 2937-2944. [CrossRef] [PubMed]

32. Zou, Y.; Huang, M.; Wang, K.; Song, B.; Wang, Y.; Chen, J.; Liu, X.; Li, X.; Lin, L.; Huang, G. Urine Surface-Enhanced Raman spectroscopy for Non-Invasive diabetic detection based on a portable Raman spectrometer. Laser Phys. Lett. 2016, 13. [CrossRef]

33. Moreira, L.P.; Silveira, L., Jr.; da Silva, A.G.; Fernandes, A.B.; Pacheco, M.T.T.; Rocco, D. Raman spectroscopy applied to identify metabolites in urine of physically active subjects. J. Photochem. Photobiol. B 2017, 176, 92-99. [CrossRef] [PubMed]

(C) 2019 by the authors. Licensee MDPI, Basel, Switzerland. This article is an open access article distributed under the terms and conditions of the Creative Commons Attribution (CC BY) license (http://creativecommons.org/licenses/by/4.0/). 